\title{
Exogenous application of glycinebetaine and potassium for improving water relations and grain yield of wheat under drought
}

\author{
M.A.S. Raza ${ }^{1 *}$, M.F. Saleem², G.M. Shah ${ }^{3,4}$, I.H. Khan ${ }^{5}$ and A. Raza ${ }^{6}$ \\ ${ }^{1}$ University College of Agriculture \& Environmental Sciences, The Islamia University of Bahawalpur, Pakistan. ${ }^{2}$ Department \\ of Agronomy, University of Agriculture, Faisalabad, Pakistan. ${ }^{3}$ Farming Systems Ecology Group, Wageningen University, The \\ Netherlands. ${ }^{4}$ Department of Environmental Sciences, COMSATS Institute of Information Technology Vehari-Pakistan. ${ }^{5}$ Department \\ of Agronomy, Dera Ghazi Khan Campus, University of Agriculture, Faisalabad, Pakistan. ${ }^{6}$ Department of Agriculture Extension and \\ Rural development, University of Agriculture Faisalabad, Pakistan. *Corresponding author : aownsamar@gmail.com.
}

\begin{abstract}
Compatible solutes rescue plants in the hour of intense water deficit conditions. Glycinebetaine (GB) and potassium $(\mathrm{K})$ are main solutes, playing role in improving plant water potential and ultimately the crop yield. However, only a few attempts have been made so far to study their optimum dozes and interactions to ameliorate the drought stress in wheat. To explore this, GB solutions of $0,50,100$ and $150 \mathrm{mM}$ concentration and $\mathrm{K}$ solutions of $0,0.5,1.0$, and $1.5 \%$ concentration were sprayed at milking stage of two wheat varieties under stress (Auqab-2000; drought sensitive and Lasani-2008; drought resistant). The stress was created by withholding water up till appearance of wilting symptoms and then the solutes (alone and/or in combination) were sprayed with carboxymethyl cellulose as a sticking agent, whereas Tween-20 was used as a surfactant for foliar spray. At maturity, ten random plants from field-experiments and three in case of pot experiment were selected to estimate plant height, spike length, number of spikelets spike ${ }^{-1}$, number of grains spike ${ }^{-1}$, and grain yields. Besides, water potential, osmotic potential and turgor potential of crop were also estimated.

The results indicated that the drought stress adversely affected all the above parameters. The exogenous application of GB and $\mathrm{K}$ to wheat significantly improved spike length, number of grain per spike and grain yields. Moreover, a significant interaction between these solutes was observed since at a given level of GB all these yield parameters were increased $(p<0.05)$ with K concentration. The highest values were obtained when GB and K were applied in combination at $100 \mathrm{mM}$ and $1.5 \%$, respectively. The same treatment also improved the leaf water potential, osmotic potential and turgor potential to maintain plant water potential gradient under stress. These findings lead us to conclude that application of GB and $\mathrm{K}(100 \mathrm{mM}$ and $1.5 \%$, respectively) is the best strategy to ameliorate the drought impact on wheat at milking stage with improved production.
\end{abstract}

Keywords: Foliar spray, drought tolerance, leaf water potential, water deficit, wheat yield 


\section{Introduction}

One third of the world's population consumes wheat (Triticum aestivum L.) as staple food because it is one of the main sources of carbohydrates and nutrition. It provides calories in the form of starch, proteins and vitamin I. In Pakistan, wheat is grown on an area of 8666 thousand hectares and production is 23.5 million tones with an average yield of $2714 \mathrm{~kg} \mathrm{ha}^{-1}$ (Govt. of Pakistan 2012). These yields are far less than the genetic yielding potential of wheat varieties cultivated in the area. One of the reasons for the reduced crop yields is unavailability of irrigation water due to low rainfall, low river flow, depletion of ground water and decreased water reservoir capacities (Govt. of Pakistan 2012) which lead to drought stress and thereby the reduction in crop yield (Raza et al. 2012).

Water is essential at every stage of plant growth from seed germination to plant maturation and seed development. Therefore, drought stress affects the crop yield regardless of the growth stage at which it occurs. However, on critical growth stages the damaging effect of water deficit reduced crop yield depending upon the genotypes. In this response they show many morphological and physiological alterations under unfavorable situations (Sakamoto and Murata 2002). Accumulation of certain low molecular weight organic metabolites such as quaternary ammonium compounds is important mechanism in response to drought stress in plants. The organic solutes are harmless to cellular functions, even at high concentrations (Serraj and Sinclair 2002).

Though many techniques have been practiced on plants to cope with drought stress but exogenous application of compatible solutes including sugar, polyols, amino acids, proline and glycinebetaine, etc. are getting important consideration in modern agricultural research. Accumulation of these compounds endure the plants to retain water within cells and protect cellular compartments from injury caused by dehydration and thus balancing turgor pressure, during drought stress (Bohnert et al., 1995). Application of glycinebetaine has been reported to enhance germination and seedling growth of plants under stressful environment (Gadallah 1999). It has also been used to enhance the drought tolerance of some crops species like wheat (Allard et al. 1998) rice (Rahman et al., 2002) and cotton (Naidu et al. 1998). Moreover, potassium application has demonstrated increase in number of fertile tillers (Mehdi et al. 2001), biomass production (Akhtar et al. 2002), number of grains per ear (Evans and Riedell 2006), 1000 -grain weight and grain yield (Evans and Riedell 2006). Potassium increases stress tolerance in field crops by improving the water contents in leaves (Thalooth et al. 1990). It is one of the essential elements and is required by the plant in large quantities for maintaining the osmotic balance and opening and closing of stomata; enzymes like the pyruvate kinase uses potassium as cofactor. Potassium is a major osmotically active solute of plant cell (Mengel and Arncke 1982) and adjusts superoxide dismutase (SOD) activity in plants, mitigate the injury of active oxygen derived from drought stress to plasma membrane and maintain the integrality of cell membrane, protecting enzyme activity in cells. Thus drought resistance of the plant could be strengthened by potassium.

The bottom lines of the reviewed results in this section indicate that under drought stress conditions, yield losses can be minimized by the application of solutes such as potassium and glycinebetain. However, their optimum dosages and interactions are not well understood yet. Thus the objective of the study is to investigate the effect of various dosages of potassium and glycinebetain, and their interaction in ameliorating the drought stress in wheat.

\section{Materials and Methods}

To explore the objective as outlined above, an outdoor pot experiment and two field experiments were conducted at Nuclear Institute for Agriculture and Biology, Faisalabad, Pakistan or University of Agriculture, Faisalabad, Pakistan (31.430 N, 73.080 E at 184.4 masl). 


\subsection{Experimental layout and treatments}

\subsubsection{Experimental layout}

Pot experiment: The pot experiment was conducted in a wire house in order to avoid any (i) insect/birds attack on the plants and (ii) interruption by rainfall. The latter was done by covering the wire house structure with polyethylene plastic sheet at the time of rainfall. Physico-chemical analysis of the experimental soil showed that it contained; sand $22 \%$, silt $13 \%$, clay $65 \%$, organic matter $0.83 \%$, total nitrogen $(\mathrm{N}) 0.33 \mathrm{mg}$ $\mathrm{kg}^{-1}$ dry soil, phosphorous (P) $4.9 \mathrm{mg} \mathrm{kg}^{-1}$ dry soil, $\mathrm{K}$ $128 \mathrm{mg} \mathrm{kg}^{-1}$ dry soil, and calcium (Ca) $101 \mathrm{mg} \mathrm{kg}^{-1}$ dry soil. The $\mathrm{pH}$ of the soil was 7.7

The soil was filled in pots with amount of $7 \mathrm{~kg}$ dry soil per pot and subsequently the recommended doses of $\mathrm{N}$ and $\mathrm{P}\left(120 \mathrm{~g} \mathrm{pot}^{-1}\right)$ was applied. Thereafter, two commonly grown wheat cultivars (Lasani-2008 and Auqab-2000) in Pakistan were sown at a rate of 10 seeds per pot. After 14 days of germination, plants were thinned to four plants per pot. During the growth period, drought stress was created at milking stage by withholding irrigation till the appearance of wilting symptoms.

Field experiment: Two field experiments were conducted during 2009 and 2010. For this purpose seeds at a rate of $40 \mathrm{~kg} \mathrm{ha}^{-1}$ were sown on 11-11-2009 at Nuclear Institute for Agriculture and Biology (NIAB), Faisalabad, Pakistan and on 17-11-2010 at University of Agriculture Faisalabad, Pakistan during 2010-11. In both cases net plot size was $4 \mathrm{~m} \times 1.2 \mathrm{~m}$ and 5 lines per plot were maintained. The recommended doses of $\mathrm{N}$ and $\mathrm{P}$ were applied during soil preparation. Experiments were laid out following randomized complete block design (RCBD) in the field with factorial arrangement and three replications. The drought stress was created at grain filling stage by withholding irrigation similar to that of pot experiment.

\subsubsection{Experimental treatments}

After creating drought stress at milking stage wheat grown of both in pots and the field, four levels of GB
$(0,50,100$ and $150 \mathrm{mM})$ and $\mathrm{K}(0.0 .5,1$ and $1.5 \%)$ were sprayed, 70 days after sowing of wheat in pots and 73 days after sowing in field. For the foliar spray carboxymethyl cellulose ( $5 \%$ solution) was used as a sticking agent, whereas Tween-20 ( $0.1 \%$ solution $)$ was used as a surfactant. The control plot or pots were also included with similar agronomic practices but without GB or K application, however, the carrier solution of carboxymethyle cellulose and tween-20 was applied.

\subsection{Data collection and analysis}

\subsubsection{Growth and yield parameters}

At maturity, ten random plants from each plot from field experiments and three plants from each pot in case of pot experiment were selected to estimate the plant height, spike length, number of spikelets spike ${ }^{-1}$, number of grains spike $e^{-1}$, and grain yields.

\subsubsection{Water relations measurements}

The fully expanded youngest leaf of two plants of each treatment was used to determine the leaf water potential. The measurements were made from 8.00 to 10.00 a.m. with Scholander type pressure chamber. The leaf, which was used for water potential, was frozen in a freezer below $-20{ }^{\circ} \mathrm{C}$ for seven days. The frozen leaf material was then thawed and cell sap extracted with the help of a disposable syringe. The sap so extracted was directly used for the determination of osmotic potential using an osmometer (Wescor 5500). The turgor potential $\left(\Psi_{\mathrm{p}}\right)$ was calculated using the equation $\left(\Psi_{\mathrm{p}}\right)=\left(\Psi_{\mathrm{w}}\right)-\left(\Psi_{\mathrm{s}}\right)$. Where $\left(\Psi_{\mathrm{w}}\right)$ is water potential and $\left(\Psi_{\mathrm{s}}\right)$ is osmotic potential.

\subsubsection{Statistical analysis}

Data collected was statistically analyzed using analysis of variance technique in Statistix (8th edition, Analytical Software, USA) and treatment means were compared by Fisher's least significant test at 5\% probability (Steel et al. 1997). 


\section{Results}

The results of plant height are presented in Table 1 . We found a significant difference between the varieties since plant height was higher in Lasani-2008 than in Auqab-2000. Wheat plants gave significant response to different levels of GB application both in pots as well as in field. Among all the GB application levels, treatment $T_{2}$ had the highest plant height as compared to the others. Significant response was also noted with different levels of $\mathrm{K}$ application. Among these, the treatment $\mathrm{K}_{3}$ had highest plant height, and was at par with $\mathrm{K}_{2}$. Furthermore, interactive effect of exogenous applications of $\mathrm{GB}$ and $\mathrm{K}(\mathrm{T} \times \mathrm{K})$ was non-significant on plant height of wheat (Table 1). Nevertheless, the treatment $\mathrm{T}_{2} \mathrm{~K}_{3}$ had the highest plant height, whereas the lowest values were observed in case of $\mathrm{T}_{0} \mathrm{~K}_{0}$. Overall, plant height was higher in 2010-11 than 2009-10 (Table 1). Interestingly, for all the measured parameters no interactions were observed (i) between the GB and wheat varieties, (ii) between the $\mathrm{K}$ and wheat varieties and (iii) among the GB, $\mathrm{K}$ and wheat variety. Therefore, in the next paragraphs this affect will not be discussed.

Data on spike length in Table 2 showed that varieties behaved significantly both in pots as well as in field (2009-10 and 2010-11). Lasani-2008 had significantly more spike length as compared to Auqab-2000. In the three experiments different GB levels significantly increased the spike length as compared to the control where no GB or $\mathrm{K}$ was applied. Among $\mathrm{K}$ rates the maximum spike length was with observed in $\mathrm{K}_{3}$. In all the three experiments interactions between GB and $\mathrm{K}$ applications was also significant. Therefore, the highest spike length was obtained in $\mathrm{T}_{2} \mathrm{~K}_{3}$. Minimum spike length was obtained in $\mathrm{T}_{0} \mathrm{~K}_{0}$.

Number of spikelets per spike significantly $(p<$ 0.05 ) affected by the varieties and application of GB and $\mathrm{K}$. (Table 3). Between the varieties, on average spikelets per spike were higher from Lasani 2008 than Auqab-2000. In case of GB applications, the highest number of spikelets per spike was achieved at $T_{2}$ whilst the minimum was obtained on plants under drought and without GB or K application. From K treatments these numbers was the highest at $\mathrm{K}_{3}$.

The average numbers of grains per spike are presented in Table 4. The results showed that both varieties differed significantly, and Lasani-2008 had more grain per spike. Application of GB at different dosages affected significantly the number of grains. The highest number of grains was achieved in $\mathrm{T}_{2}$. A significant decrease in grains per spike recorded when stress was imposed on the plants without spray of GB. Foliar application of $\mathrm{K}$ effect was also significant on grains per spike. The maximum grain number was obtained in $\mathrm{K}_{3}$ and the lowest in $\mathrm{K}_{0}$. Interaction between the $\mathrm{GB}$ and $\mathrm{K}$ application was also significant both in pots as well as in field. More grains per spike was obtained in $\mathrm{T}_{2} \mathrm{~K}_{3}$, it was at par with $\mathrm{T}_{2} \mathrm{~K}_{2}$, while minimum spike length was obtained in $\mathrm{T}_{0} \mathrm{~K}_{0}$ both in pots as well as in the field. Number of grains per spike was higher in 2010-11 than in 2009-10.

Table 5 shows the average gain yield from both the pot and field experiment. The results indicate that the grain yield was significantly higher from Lasani 2008 than Auqab 2000. Application of GB and K to plants under drought stress significantly improved the yield as compared to the plants receiving no GB or K. Among different GB application doses highest grain yield was obtained in treatment $T_{2}$, and produced minimum grain yield in treatment $T_{0}$. Also the foliar application of $\mathrm{K}$ significantly improved the grain yield per plant. More grain yield was produced when crop was sprayed with solution of higher $\mathrm{K}$ concentration (1.5\%) in both trials (pot/field); however, in pots trial it was at par with that of $\mathrm{K}$ solution of $1 \%$, while lowest grain yield was obtained in treatment $\mathrm{K}_{0}$. Interaction between $\mathrm{GB}$ and $\mathrm{K}$ was also significant both in pots and in field (200910 and 2010-11). The highest grain yield was achieved in treatment $\mathrm{T}_{2} \mathrm{~K}_{3}$ in all the three trials; however, in pots trial $\mathrm{T}_{2} \mathrm{~K}_{3}$ was at par with $\mathrm{T}_{1} \mathrm{~K}_{2}$, whilst the lower grain yield was observed with $\mathrm{T}_{0} \mathrm{~K}_{0}$ (Table 5). Grain yield was more in 2010-11 than 2009-10. 
Table 1. Average plant height $(\mathrm{cm})$ of wheat influenced by the application of glycinebetaine $(\mathrm{GB})$ and potassium $(\mathrm{K})$ in three experiments. Means with different letters are significantly different from each other at $p \geq 0.05$.

\begin{tabular}{|c|c|c|c|}
\hline \multirow{2}{*}{ Factors } & \multicolumn{3}{|c|}{ Experiments } \\
\hline & Pots trial & Field trial 2009-10 & Field trial 2010-11 \\
\hline \multicolumn{4}{|l|}{ Varieties } \\
\hline $\mathrm{V}_{1}=$ Lasani-2008 & $77.2 \mathrm{a}$ & $83.4 \mathrm{a}$ & $85.4 \mathrm{a}$ \\
\hline $\mathrm{V}_{2}=$ Auqab -2000 & $74.2 \mathrm{~b}$ & $79.3 \mathrm{~b}$ & $82.1 \mathrm{~b}$ \\
\hline \multicolumn{4}{|l|}{ GB application rate (T) } \\
\hline $\mathrm{T}_{0}=0 \%$ & $72.1 \mathrm{c}$ & $77.8 \mathrm{c}$ & $80.5 \mathrm{~d}$ \\
\hline $\mathrm{T}_{1}=50 \mathrm{mM}$ & $77.1 \mathrm{ab}$ & $82.6 \mathrm{ab}$ & $84.4 \mathrm{~b}$ \\
\hline $\mathrm{T}_{2}=100 \mathrm{mM}$ & $78.2 \mathrm{a}$ & $84.1 \mathrm{a}$ & $86.6 \mathrm{a}$ \\
\hline $\mathrm{T}_{3}=150 \mathrm{mM}$ & $75.3 \mathrm{~b}$ & $80.7 \mathrm{~b}$ & $83.3 \mathrm{c}$ \\
\hline \multicolumn{4}{|l|}{ K application rate $(\mathrm{K})$} \\
\hline $\mathrm{K}_{0=} 0 \%$ & $70.3 \mathrm{c}$ & $75.8 \mathrm{c}$ & $80.7 \mathrm{~d}$ \\
\hline $\mathrm{K}_{1=0.5 \%}$ & $74.2 \mathrm{~b}$ & $80.1 \mathrm{~b}$ & $82.7 \mathrm{c}$ \\
\hline $\mathrm{K}_{2}=1.0 \%$ & $78.4 \mathrm{a}$ & $84.0 \mathrm{a}$ & $84.9 \mathrm{~b}$ \\
\hline $\mathrm{K}_{3}=1.5 \%$ & $79.8 \mathrm{a}$ & $85.3 \mathrm{a}$ & $86.6 \mathrm{a}$ \\
\hline \multicolumn{4}{|l|}{ T x K } \\
\hline $\mathrm{T}_{0} \mathrm{~K}_{0}=0 \% \mathrm{~GB}+0 \% \mathrm{~K}$ & $68.7 \mathrm{a}$ & $74.7 \mathrm{a}$ & $78.0 \mathrm{a}$ \\
\hline $\mathrm{T}_{0} \mathrm{~K}_{1}=0 \% \mathrm{~GB}+0.5 \% \mathrm{~K}$ & $70.8 \mathrm{a}$ & $76.3 \mathrm{a}$ & $79.5 \mathrm{a}$ \\
\hline $\mathrm{T}_{0} \mathrm{~K}_{2=}=\% \mathrm{~GB}+1 \% \mathrm{~K}$ & $74.0 \mathrm{a}$ & $79.8 \mathrm{a}$ & $81.5 \mathrm{a}$ \\
\hline $\mathrm{T}_{0} \mathrm{~K}_{3}=0 \% \mathrm{~GB}+1.5 \% \mathrm{~K}$ & $75.0 \mathrm{a}$ & $80.5 \mathrm{a}$ & $83.2 \mathrm{a}$ \\
\hline $\mathrm{T}_{1} \mathrm{~K}_{0=} 50 \mathrm{mM} \mathrm{GB}+0 \% \mathrm{~K}$ & $72.0 \mathrm{a}$ & $77.5 \mathrm{a}$ & $81.0 \mathrm{a}$ \\
\hline $\mathrm{T}_{1} \mathrm{~K}_{\mathrm{l}}=50 \mathrm{mM} \mathrm{GB}+0.5 \% \mathrm{~K}$ & $75.2 \mathrm{a}$ & $80.7 \mathrm{a}$ & $83.8 \mathrm{a}$ \\
\hline $\mathrm{T}_{1} \mathrm{~K}_{2}=50 \mathrm{mM} \mathrm{GB}+1 \% \mathrm{~K}$ & $79.8 \mathrm{a}$ & $85.3 \mathrm{a}$ & $85.8 \mathrm{a}$ \\
\hline $\mathrm{T}_{1} \mathrm{~K}_{3=} 50 \mathrm{mM} \mathrm{GB}+1.5 \% \mathrm{~K}$ & $81.7 \mathrm{a}$ & $87.2 \mathrm{a}$ & $87.0 \mathrm{a}$ \\
\hline $\mathrm{T}_{2} \mathrm{~K}_{0=}=100 \mathrm{mM} \mathrm{GB}+0 \% \mathrm{~K}$ & $72.0 \mathrm{a}$ & $77.3 \mathrm{a}$ & $83.3 \mathrm{a}$ \\
\hline $\mathrm{T}_{2} \mathrm{~K}_{1}=100 \mathrm{mM} \mathrm{GB}+0.5 \% \mathrm{~K}$ & $77.7 \mathrm{a}$ & $84.8 \mathrm{a}$ & $85.3 \mathrm{a}$ \\
\hline $\mathrm{T}_{2} \mathrm{~K}_{2}=100 \mathrm{mM} \mathrm{GB}+1 \% \mathrm{~K}$ & $80.8 \mathrm{a}$ & $86.3 \mathrm{a}$ & $87.5 \mathrm{a}$ \\
\hline $\mathrm{T}_{2} \mathrm{~K}_{3}=100 \mathrm{mM} \mathrm{GB}+1.5 \% \mathrm{~K}$ & $82.5 \mathrm{a}$ & $88.0 \mathrm{a}$ & $90.3 \mathrm{a}$ \\
\hline $\mathrm{T}_{3} \mathrm{~K}_{0}=150 \mathrm{mM} \mathrm{GB}+0 \% \mathrm{~K}$ & $68.2 \mathrm{a}$ & $73.8 \mathrm{a}$ & $80.5 \mathrm{a}$ \\
\hline $\mathrm{T}_{3} \mathrm{~K}_{1}=150 \mathrm{mM} \mathrm{GB}+0.5 \% \mathrm{~K}$ & $73.2 \mathrm{a}$ & $78.7 \mathrm{a}$ & $82.2 \mathrm{a}$ \\
\hline $\mathrm{T}_{3} \mathrm{~K}_{2}=150 \mathrm{mM} \mathrm{GB}+1 \% \mathrm{~K}$ & $79.0 \mathrm{a}$ & $84.5 \mathrm{a}$ & $84.8 \mathrm{a}$ \\
\hline $\mathrm{T}_{3} \mathrm{~K}_{3}=150 \mathrm{mM} \mathrm{GB}+1.5 \% \mathrm{~K}$ & $80.3 \mathrm{a}$ & $85.8 \mathrm{a}$ & $86.0 \mathrm{a}$ \\
\hline
\end{tabular}


Table 2. Average spike length $(\mathrm{cm})$ of wheat as influenced by the application of glycinebetaine $(\mathrm{GB})$ and potassium $(\mathrm{K})$ in the three experiments. Means with different letters are significantly different from each other at $p \geq 0.05$.

\begin{tabular}{|c|c|c|c|}
\hline \multirow[t]{2}{*}{ Factors } & \multicolumn{3}{|c|}{ Experiments } \\
\hline & Pots trial & Field trial 2009-10 & Field trial 2010-11 \\
\hline $\mathrm{V}_{1=}$ Lasani-2008 & $9.0 \mathrm{a}$ & $10.7 \mathrm{a}$ & $13.9 \mathrm{a}$ \\
\hline $\mathrm{V}_{2}=$ Auqab-2000 & $8.8 \mathrm{~b}$ & $9.03 \mathrm{~b}$ & $12.6 \mathrm{~b}$ \\
\hline \multicolumn{4}{|l|}{ GB application rate $(\mathrm{T})$} \\
\hline $\mathrm{T}_{0}=0 \%$ & $8.5 \mathrm{c}$ & $9.5 \mathrm{c}$ & $10.7 \mathrm{~d}$ \\
\hline $\mathrm{T}_{1}=50 \mathrm{mM}$ & $8.8 \mathrm{~b}$ & $9.8 \mathrm{~b}$ & $13.0 \mathrm{c}$ \\
\hline $\mathrm{T}_{2=}=100 \mathrm{mM}$ & $9.7 \mathrm{a}$ & $10.5 \mathrm{a}$ & $15.1 \mathrm{a}$ \\
\hline $\mathrm{T}_{3}=150 \mathrm{mM}$ & $8.5 \mathrm{c}$ & $9.6 \mathrm{c}$ & $14.2 \mathrm{~b}$ \\
\hline \multicolumn{4}{|l|}{$\mathrm{K}$ application rate $(\mathrm{K})$} \\
\hline $\mathrm{K}_{0=} 0 \%$ & $8.4 \mathrm{c}$ & $9.4 \mathrm{c}$ & $11.9 \mathrm{~d}$ \\
\hline $\mathrm{K}_{1=} 0.5 \%$ & $8.8 \mathrm{~b}$ & $9.8 \mathrm{~b}$ & $12.8 \mathrm{c}$ \\
\hline $\mathrm{K}_{2}=1.0 \%$ & $9.1 \mathrm{a}$ & $10.1 \mathrm{a}$ & $13.8 \mathrm{~b}$ \\
\hline $\mathrm{K}_{3}=1.5 \%$ & $9.2 \mathrm{a}$ & $10.2 \mathrm{a}$ & $14.5 \mathrm{a}$ \\
\hline \multicolumn{4}{|l|}{$\mathrm{T} \times \mathrm{K}$} \\
\hline $\mathrm{T}_{0} \mathrm{~K}_{0}=0 \% \mathrm{~GB}+0 \% \mathrm{~K}$ & $7.7 \mathrm{~g}$ & $9.4 \mathrm{~d}$ & $9.2 \mathrm{i}$ \\
\hline $\mathrm{T}_{0} \mathrm{~K}_{1}=0 \% \mathrm{~GB}+0.5 \% \mathrm{~K}$ & $8.5 \mathrm{ef}$ & $9.5 \mathrm{~d}$ & $10.8 \mathrm{~h}$ \\
\hline $\mathrm{T}_{0} \mathrm{~K}_{2}=0 \% \mathrm{~GB}+1 \% \mathrm{~K}$ & $8.8 \mathrm{de}$ & $9.6 \mathrm{~d}$ & $11.3 \mathrm{gh}$ \\
\hline $\mathrm{T}_{0} \mathrm{~K}_{3}=0 \% \mathrm{~GB}+1.5 \% \mathrm{~K}$ & $9.0 \mathrm{~cd}$ & $9.6 \mathrm{~d}$ & $11.6 \mathrm{~g}$ \\
\hline $\mathrm{T}_{1} \mathrm{~K}_{0=} 50 \mathrm{mM} \mathrm{GB}+0 \% \mathrm{~K}$ & 8.5 ef & $9.4 \mathrm{~d}$ & $11.3 \mathrm{gh}$ \\
\hline $\mathrm{T}_{1} \mathrm{~K}_{1}=50 \mathrm{mM} \mathrm{GB}+0.5 \% \mathrm{~K}$ & $8.8 \mathrm{de}$ & $9.7 \mathrm{~cd}$ & $11.8 \mathrm{~g}$ \\
\hline $\mathrm{T}_{1} \mathrm{~K}_{2}=50 \mathrm{mM} \mathrm{GB}+1 \% \mathrm{~K}$ & $8.9 \mathrm{~cd}$ & $12.1 \mathrm{~b}$ & $14.2 \mathrm{de}$ \\
\hline $\mathrm{T}_{1} \mathrm{~K}_{3}=50 \mathrm{mM} \mathrm{GB}+1.5 \% \mathrm{~K}$ & $9.2 \mathrm{bc}$ & $12.2 \mathrm{~b}$ & $14.7 \mathrm{~cd}$ \\
\hline $\mathrm{T}_{2} \mathrm{~K}_{0=}=100 \mathrm{mM} \mathrm{GB}+0 \% \mathrm{~K}$ & $9.2 \mathrm{bc}$ & $12.2 \mathrm{~b}$ & $13.8 \mathrm{ef}$ \\
\hline $\mathrm{T}_{2} \mathrm{~K}_{1}=100 \mathrm{mM} \mathrm{GB}+0.5 \% \mathrm{~K}$ & $9.5 \mathrm{~b}$ & $12.3 \mathrm{~b}$ & $14.5 \mathrm{ce}$ \\
\hline $\mathrm{T}_{2} \mathrm{~K}_{2}=100 \mathrm{mM} \mathrm{GB}+1 \% \mathrm{~K}$ & $10.0 \mathrm{a}$ & $12.7 \mathrm{a}$ & $15.4 \mathrm{~b}$ \\
\hline $\mathrm{T}_{2} \mathrm{~K}_{3=} 100 \mathrm{mM} \mathrm{GB}+1.5 \% \mathrm{~K}$ & $10.2 \mathrm{a}$ & $12.89 \mathrm{a}$ & $16.8 \mathrm{a}$ \\
\hline $\mathrm{T}_{3} \mathrm{~K}_{0}=150 \mathrm{mM} \mathrm{GB}+0 \% \mathrm{~K}$ & $8.4 \mathrm{f}$ & $10.6 \mathrm{e}$ & $13.5 \mathrm{f}$ \\
\hline $\mathrm{T}_{3} \mathrm{~K}_{1=} 150 \mathrm{mM} \mathrm{GB}+0.5 \% \mathrm{~K}$ & 8.4 ef & $11.5 \mathrm{~d}$ & $14.0 \mathrm{~d}-\mathrm{f}$ \\
\hline $\mathrm{T}_{3} \mathrm{~K}_{2=}=150 \mathrm{mM} \mathrm{GB}+1 \% \mathrm{~K}$ & 8.6 ef & $12.0 \mathrm{bc}$ & 14.4 c-e \\
\hline $\mathrm{T}_{3} \mathrm{~K}_{3}=150 \mathrm{mM} \mathrm{GB}+1.5 \% \mathrm{~K}$ & $8.6 \mathrm{~d}-\mathrm{f}$ & $12.3 \mathrm{~b}$ & $15.1 \mathrm{bc}$ \\
\hline $\mathrm{V} \times \mathrm{T}, \mathrm{V} \times \mathrm{K}, \mathrm{V} \times \mathrm{T} \times \mathrm{K}$ & Non significant & & \\
\hline
\end{tabular}


Table 3. Average number of spikelets per spike of wheat influenced by the application of glycinebetaine (GB) and potassium $(\mathrm{K})$ under drought in natural conditions. Means with different letters are significantly different from each other at $p \geq 0.05$.

\begin{tabular}{|c|c|c|c|}
\hline \multirow[t]{2}{*}{ Factors } & \multicolumn{3}{|c|}{ Experiments } \\
\hline & Pots trial & Field trial 2009-10 & Field trial 2010-11 \\
\hline $\mathrm{V}_{1=}$ Lasani-2008 & $15.4 \mathrm{a}$ & $17.5 \mathrm{a}$ & $18.3 \mathrm{a}$ \\
\hline $\mathrm{V}_{2}=$ Auqab-2000 & $14.3 \mathrm{~b}$ & $15.4 \mathrm{~b}$ & $17.1 \mathrm{~b}$ \\
\hline \multicolumn{4}{|l|}{ GB application rate $(\mathrm{T})$} \\
\hline $\mathrm{T}_{0=}=0 \%$ & $13.8 \mathrm{~d}$ & $16.0 \mathrm{c}$ & $15.0 \mathrm{~d}$ \\
\hline $\mathrm{T}_{1}=50 \mathrm{mM}$ & $14.7 \mathrm{~b}$ & $16.5 \mathrm{~b}$ & $17.5 \mathrm{c}$ \\
\hline $\mathrm{T}_{2}=100 \mathrm{mM}$ & $16.3 \mathrm{a}$ & $17.1 \mathrm{a}$ & $19.7 \mathrm{a}$ \\
\hline $\mathrm{T}_{3}=150 \mathrm{mM}$ & $14.6 \mathrm{c}$ & $16.4 \mathrm{~b}$ & $18.6 \mathrm{~b}$ \\
\hline \multicolumn{4}{|l|}{$\mathrm{K}$ application rate $(\mathrm{K})$} \\
\hline $\mathrm{K}_{0=0} \%$ & $14.0 \mathrm{c}$ & $15.8 \mathrm{c}$ & $15.9 \mathrm{~d}$ \\
\hline $\mathrm{K}_{1}=0.5 \%$ & $14.4 \mathrm{~b}$ & $16.2 \mathrm{~b}$ & $17.1 \mathrm{c}$ \\
\hline $\mathrm{K}_{2}=1.0 \%$ & $15.6 \mathrm{a}$ & $17.0 \mathrm{a}$ & $18.3 \mathrm{~b}$ \\
\hline $\mathrm{K}_{3}=1.5 \%$ & $15.5 \mathrm{a}$ & $16.9 \mathrm{a}$ & $19.6 \mathrm{a}$ \\
\hline \multicolumn{4}{|l|}{$\mathrm{T} \times \mathrm{K}$} \\
\hline $\mathrm{T}_{0} \mathrm{~K}_{0}=0 \% \mathrm{~GB}+0 \% \mathrm{~K}$ & $13.1 \mathrm{k}$ & $15.7 \mathrm{e}$ & 13.21 \\
\hline $\mathrm{T}_{0} \mathrm{~K}_{1=}=0 \mathrm{~GB}+0.5 \% \mathrm{~K}$ & $13.6 \mathrm{ij}$ & $15.7 \mathrm{e}$ & $14.8 \mathrm{k}$ \\
\hline $\mathrm{T}_{0} \mathrm{~K}_{2}=0 \% \mathrm{~GB}+1 \% \mathrm{~K}$ & $14.4 \mathrm{f}$ & $16.2 \mathrm{~cd}$ & $15.7 \mathrm{jk}$ \\
\hline $\mathrm{T}_{0} \mathrm{~K}_{3}=0 \% \mathrm{~GB}+1.5 \% \mathrm{~K}$ & $14.3 \mathrm{f}$ & $16.2 \mathrm{~d}$ & $16.3 \mathrm{ij}$ \\
\hline $\mathrm{T}_{1} \mathrm{~K}_{0=} 50 \mathrm{mM} \mathrm{GB}+0 \% \mathrm{~K}$ & $13.8 \mathrm{gh}$ & $15.7 \mathrm{e}$ & $16.0 \mathrm{ij}$ \\
\hline $\mathrm{T}_{1} \mathrm{~K}_{1}=50 \mathrm{mM} \mathrm{GB}+0.5 \% \mathrm{~K}$ & $13.9 \mathrm{~g}$ & $15.8 \mathrm{e}$ & $16.3 \mathrm{~g}-\mathrm{i}$ \\
\hline $\mathrm{T}_{1} \mathrm{~K}_{2}=50 \mathrm{mM} \mathrm{GB}+1 \% \mathrm{~K}$ & $15.7 \mathrm{~cd}$ & $17.3 \mathrm{ab}$ & $18.0 \mathrm{ef}$ \\
\hline $\mathrm{T}_{1} \mathrm{~K}_{3}=50 \mathrm{mM} \mathrm{GB}+1.5 \% \mathrm{~K}$ & $15.6 \mathrm{ce}$ & $17.2 \mathrm{ab}$ & $19.2 \mathrm{~cd}$ \\
\hline $\mathrm{T}_{2} \mathrm{~K}_{0=} 100 \mathrm{mM} \mathrm{GB}+0 \% \mathrm{~K}$ & $15.7 \mathrm{c}$ & $16.5 \mathrm{c}$ & $17.5 \mathrm{fh}$ \\
\hline $\mathrm{T}_{2} \mathrm{~K}_{1}=100 \mathrm{mM} \mathrm{GB}+0.5 \% \mathrm{~K}$ & $16.3 \mathrm{~b}$ & $17.0 \mathrm{~b}$ & $18.8 \mathrm{de}$ \\
\hline $\mathrm{T}_{2} \mathrm{~K}_{2}=100 \mathrm{mM} \mathrm{GB}+1 \% \mathrm{~K}$ & $16.7 \mathrm{a}$ & $17.4 \mathrm{a}$ & $20.2 \mathrm{bc}$ \\
\hline $\mathrm{T}_{2} \mathrm{~K}_{3}=100 \mathrm{mM} \mathrm{GB}+1.5 \% \mathrm{~K}$ & $16.7 \mathrm{a}$ & $17.3 \mathrm{ab}$ & $22.2 \mathrm{a}$ \\
\hline $\mathrm{T}_{3} \mathrm{~K}_{0}=150 \mathrm{mM} \mathrm{GB}+0 \% \mathrm{~K}$ & $13.6 \mathrm{j}$ & $15.2 \mathrm{f}$ & $16.7 \mathrm{hj}$ \\
\hline $\mathrm{T}_{3} \mathrm{~K}_{1}=150 \mathrm{mM} \mathrm{GB}+0.5 \% \mathrm{~K}$ & $13.8 \mathrm{hi}$ & $16.2 \mathrm{~d}$ & $17.8 \mathrm{eg}$ \\
\hline $\mathrm{T}_{3} \mathrm{~K}_{2}=150 \mathrm{mM} \mathrm{GB}+1 \% \mathrm{~K}$ & $15.5 \mathrm{de}$ & $17.2 \mathrm{ab}$ & $19.3 \mathrm{~cd}$ \\
\hline $\mathrm{T}_{3} \mathrm{~K}_{3}=150 \mathrm{mM} \mathrm{GB}+1.5 \% \mathrm{~K}$ & $15.4 \mathrm{e}$ & $17.1 \mathrm{ab}$ & $20.7 \mathrm{~b}$ \\
\hline $\mathrm{V} \times \mathrm{T}, \mathrm{V} \times \mathrm{K}, \mathrm{V} \times \mathrm{T} \times \mathrm{K}$ & \multicolumn{3}{|c|}{ Non significant } \\
\hline
\end{tabular}


Table 4. Average number of grains per spike of wheat influenced by the application of glycinebetaine (GB) and potassium $(\mathrm{K})$ under drought in natural conditions. Means with different letters are significantly different from each other at $p \geq 0.05$.

\begin{tabular}{|c|c|c|c|}
\hline \multirow[t]{2}{*}{ Factors } & \multicolumn{3}{|c|}{ Experiments } \\
\hline & Pots trial & Field trial 2009-10 & Field trial 2010-11 \\
\hline $\mathrm{V}_{1}=$ Lasani-2008 & $33.3 \mathrm{a}$ & $46.8 \mathrm{a}$ & $61.0 \mathrm{a}$ \\
\hline$V_{2}=$ Auqab -2000 & $30.0 \mathrm{~b}$ & $43.2 \mathrm{~b}$ & $54.6 \mathrm{~b}$ \\
\hline \multicolumn{4}{|l|}{ GB application rate $(\mathrm{T})$} \\
\hline $\mathrm{T}_{0}=0 \%$ & $28.9 \mathrm{~d}$ & $42.6 \mathrm{c}$ & $43.0 \mathrm{~d}$ \\
\hline $\mathrm{T}_{1}=50 \mathrm{mM}$ & $31.6 \mathrm{~b}$ & $44.7 \mathrm{~b}$ & $58.9 \mathrm{c}$ \\
\hline $\mathrm{T}_{2}=100 \mathrm{mM}$ & $35.5 \mathrm{a}$ & $49.7 \mathrm{a}$ & $66.8 \mathrm{a}$ \\
\hline $\mathrm{T}_{3}=150 \mathrm{mM}$ & $30.5 \mathrm{c}$ & $42.9 \mathrm{c}$ & $62.4 \mathrm{~b}$ \\
\hline \multicolumn{4}{|l|}{$\mathrm{K}$ application rate $(\mathrm{K})$} \\
\hline $\mathrm{K}_{0=}=0 \%$ & $26.3 \mathrm{c}$ & $40.5 \mathrm{c}$ & $47.4 \mathrm{~d}$ \\
\hline $\mathrm{K}_{1}=0.5 \%$ & $29.5 b$ & $43.2 \mathrm{~b}$ & $56.0 \mathrm{c}$ \\
\hline $\mathrm{K}_{2}=1.0 \%$ & $35.0 \mathrm{a}$ & $47.5 \mathrm{a}$ & $61.8 \mathrm{~b}$ \\
\hline $\mathrm{K}_{3}=1.5 \%$ & $35.6 \mathrm{a}$ & $48.7 \mathrm{a}$ & $66.0 \mathrm{a}$ \\
\hline \multicolumn{4}{|l|}{$\mathrm{T} \times \mathrm{K}$} \\
\hline $\mathrm{T}_{0} \mathrm{~K}_{0=}=0 \mathrm{~GB}+0 \% \mathrm{~K}$ & $25.0 \mathrm{f}$ & $39.0 \mathrm{~g}$ & $28.8 \mathrm{i}$ \\
\hline $\mathrm{T}_{0} \mathrm{~K}_{1=}=0 \mathrm{~GB}+0.5 \% \mathrm{~K}$ & $27.2 \mathrm{e}$ & $40.3 \mathrm{fg}$ & $41.8 \mathrm{~h}$ \\
\hline $\mathrm{T}_{0} \mathrm{~K}_{2}=0 \% \mathrm{~GB}+1 \% \mathrm{~K}$ & $31.5 \mathrm{~d}$ & $45.0 \mathrm{~cd}$ & $49.0 \mathrm{~g}$ \\
\hline $\mathrm{T}_{0} \mathrm{~K}_{3}=0 \% \mathrm{~GB}+1.5 \% \mathrm{~K}$ & $31.8 \mathrm{~d}$ & $46.0 \mathrm{bc}$ & $52.3 \mathrm{fg}$ \\
\hline $\mathrm{T}_{1} \mathrm{~K}_{0=} 50 \mathrm{mM} \mathrm{GB}+0 \% \mathrm{~K}$ & $27.5 \mathrm{e}$ & $42.0 \mathrm{ef}$ & $50.7 \mathrm{~g}$ \\
\hline $\mathrm{T}_{1} \mathrm{~K}_{1}=50 \mathrm{mM} \mathrm{GB}+0.5 \% \mathrm{~K}$ & $28.3 \mathrm{e}$ & $41.7 \mathrm{fg}$ & $55.8 \mathrm{ef}$ \\
\hline $\mathrm{T}_{1} \mathrm{~K}_{2}=50 \mathrm{mM} \mathrm{GB}+1 \% \mathrm{~K}$ & $34.8 \mathrm{bc}$ & $47.0 \mathrm{bc}$ & $60.7 \mathrm{~d}$ \\
\hline $\mathrm{T}_{1} \mathrm{~K}_{3}=50 \mathrm{mM} \mathrm{GB}+1.5 \% \mathrm{~K}$ & $35.8 \mathrm{~b}$ & $48.2 \mathrm{~b}$ & $68.5 \mathrm{ac}$ \\
\hline $\mathrm{T}_{2} \mathrm{~K}_{0=} 100 \mathrm{mM} \mathrm{GB}+0 \% \mathrm{~K}$ & $27.8 \mathrm{e}$ & $41.8 \mathrm{eg}$ & $57.8 \mathrm{de}$ \\
\hline $\mathrm{T}_{2} \mathrm{~K}_{1}=100 \mathrm{mM} \mathrm{GB}+0.5 \% \mathrm{~K}$ & $34.7 \mathrm{bc}$ & $48.7 \mathrm{~b}$ & $67.0 \mathrm{c}$ \\
\hline $\mathrm{T}_{2} \mathrm{~K}_{2}=100 \mathrm{mM} \mathrm{GB}+1 \% \mathrm{~K}$ & $39.5 \mathrm{a}$ & $53.5 \mathrm{a}$ & $70.2 \mathrm{ac}$ \\
\hline $\mathrm{T}_{2} \mathrm{~K}_{3}=100 \mathrm{mM} \mathrm{GB}+1.5 \% \mathrm{~K}$ & $40.0 \mathrm{a}$ & $54.7 \mathrm{a}$ & $72.2 \mathrm{a}$ \\
\hline $\mathrm{T}_{3} \mathrm{~K}_{0}=150 \mathrm{mM} \mathrm{GB}+0 \% \mathrm{~K}$ & $25.0 \mathrm{f}$ & $39.0 \mathrm{~g}$ & $52.2 \mathrm{fg}$ \\
\hline $\mathrm{T}_{3} \mathrm{~K}_{1}=150 \mathrm{mM} \mathrm{GB}+0.5 \% \mathrm{~K}$ & $28.0 \mathrm{e}$ & $42.2 \mathrm{df}$ & $59.3 \mathrm{de}$ \\
\hline $\mathrm{T}_{3} \mathrm{~K}_{2}=150 \mathrm{mM} \mathrm{GB}+1 \% \mathrm{~K}$ & $34.0 \mathrm{c}$ & $44.7 \mathrm{ce}$ & $67.3 \mathrm{bc}$ \\
\hline $\mathrm{T}_{3} \mathrm{~K}_{3}=150 \mathrm{mM} \mathrm{GB}+1.5 \% \mathrm{~K}$ & $34.8 \mathrm{bc}$ & $45.8 \mathrm{bc}$ & $70.8 \mathrm{ab}$ \\
\hline $\mathrm{V} \times \mathrm{T}, \mathrm{V} \times \mathrm{K}, \mathrm{V} \times \mathrm{T}$ x K & Non signif & & \\
\hline
\end{tabular}


Table 5. Average grain yield $\left(\mathrm{kg} \mathrm{ha}^{-1}\right)$ of wheat influenced by the application of glycinebetaine (GB) and potassium (K) under drought in natural conditions. Means with different letters are significantly different from each other at $p \geq 0.05$.

\begin{tabular}{|c|c|c|c|}
\hline \multirow[t]{2}{*}{ Factors } & \multicolumn{3}{|c|}{ Experiments } \\
\hline & $\begin{array}{l}\text { Pots trial } \\
\text { (g plant }^{-1} \text { ) }\end{array}$ & Field trial 2009-10 & Field trial 2010-11 \\
\hline $\mathrm{V}_{1=}$ Lasani-2008 & $1.8 \mathrm{a}$ & $4361.1 \mathrm{a}$ & $5289.5 \mathrm{a}$ \\
\hline$V_{2}=$ Auqab- 2000 & $1.7 \mathrm{~b}$ & $4172.1 \mathrm{~b}$ & $4606.9 \mathrm{~b}$ \\
\hline \multicolumn{4}{|l|}{ GB application rate $(\mathrm{T})$} \\
\hline $\mathrm{T}_{0}=0 \%$ & $1.7 \mathrm{~d}$ & $3386.5 \mathrm{~d}$ & $3756.4 \mathrm{~d}$ \\
\hline $\mathrm{T}_{1}=50 \mathrm{mM}$ & $1.8 \mathrm{~b}$ & $4563.0 \mathrm{~b}$ & $4880.9 \mathrm{c}$ \\
\hline $\mathrm{T}_{2}=100 \mathrm{mM}$ & $1.8 \mathrm{a}$ & $4637.3 \mathrm{a}$ & $5267.5 \mathrm{a}$ \\
\hline $\mathrm{T}_{3}=150 \mathrm{mM}$ & $1.7 \mathrm{c}$ & $4479.4 \mathrm{c}$ & $5041.3 \mathrm{~b}$ \\
\hline \multicolumn{4}{|l|}{$\mathrm{K}$ application rate $(\mathrm{K})$} \\
\hline $\mathrm{K}_{0=}=0 \%$ & $1.7 \mathrm{c}$ & $3826.8 \mathrm{~d}$ & $4056.1 \mathrm{~d}$ \\
\hline $\mathrm{K}_{1}=0.5 \%$ & $1.7 \mathrm{~b}$ & $4205.1 \mathrm{c}$ & $4569.8 \mathrm{c}$ \\
\hline $\mathrm{K}_{2}=1.0 \%$ & $1.8 \mathrm{a}$ & $4424.9 \mathrm{~b}$ & $5015.9 \mathrm{~b}$ \\
\hline $\mathrm{K}_{3}=1.5 \%$ & $1.8 \mathrm{a}$ & $4609.5 \mathrm{a}$ & $5304.2 \mathrm{a}$ \\
\hline \multicolumn{4}{|l|}{ Tx K } \\
\hline $\mathrm{T}_{0} \mathrm{~K}_{0}=0 \% \mathrm{~GB}+0 \% \mathrm{~K}$ & $1.6 \mathrm{j}$ & $3071.0 \mathrm{k}$ & $2671.1 \mathrm{j}$ \\
\hline $\mathrm{T}_{0} \mathrm{~K}_{1=}=0 \mathrm{~GB}+0.5 \% \mathrm{~K}$ & $1.7 \mathrm{hi}$ & $3404.2 \mathrm{j}$ & $3596.2 \mathrm{i}$ \\
\hline $\mathrm{T}_{0} \mathrm{~K}_{2=}=0 \mathrm{~GB}+1 \% \mathrm{~K}$ & $1.7 \mathrm{gh}$ & $3514.3 \mathrm{ij}$ & $4286.3 \mathrm{~h}$ \\
\hline $\mathrm{T}_{0} \mathrm{~K}_{3}=0 \% \mathrm{~GB}+1.5 \% \mathrm{~K}$ & $1.7 \mathrm{fg}$ & $3556.7 \mathrm{i}$ & $4472.0 \mathrm{gh}$ \\
\hline $\mathrm{T}_{1} \mathrm{~K}_{0=} 50 \mathrm{mM} \mathrm{GB}+0 \% \mathrm{~K}$ & $1.7 \mathrm{hi}$ & $4096.0 \mathrm{~g}$ & $4347.7 \mathrm{gh}$ \\
\hline $\mathrm{T}_{1} \mathrm{~K}_{1}=50 \mathrm{mM} \mathrm{GB}+0.5 \% \mathrm{~K}$ & $1.7 \mathrm{fg}$ & 4479.7 ef & 4684.7 ef \\
\hline $\mathrm{T}_{1} \mathrm{~K}_{2}=50 \mathrm{mM} \mathrm{GB}+1 \% \mathrm{~K}$ & $1.8 \mathrm{bc}$ & $4705.5 \mathrm{~d}$ & $5104.3 \mathrm{~d}$ \\
\hline $\mathrm{T}_{1} \mathrm{~K}_{3=} 50 \mathrm{mM} \mathrm{GB}+1.5 \% \mathrm{~K}$ & $1.8 \mathrm{~b}$ & $4971.0 \mathrm{ab}$ & $5386.8 \mathrm{bc}$ \\
\hline $\mathrm{T}_{2} \mathrm{~K}_{0=}=100 \mathrm{mM} \mathrm{GB}+0 \% \mathrm{~K}$ & $1.8 \mathrm{~cd}$ & $4209.5 \mathrm{~g}$ & $4705.2 \mathrm{e}$ \\
\hline $\mathrm{T}_{2} \mathrm{~K}_{1}=100 \mathrm{mM} \mathrm{GB}+0.5 \% \mathrm{~K}$ & $1.8 \mathrm{~b}$ & $4540.8 \mathrm{e}$ & $5121.8 \mathrm{~d}$ \\
\hline $\mathrm{T}_{2} \mathrm{~K}_{2=} 100 \mathrm{mM} \mathrm{GB}+1 \% \mathrm{~K}$ & $1.9 \mathrm{a}$ & $4758.5 \mathrm{~cd}$ & $5458.8 \mathrm{~b}$ \\
\hline $\mathrm{T}_{2} \mathrm{~K}_{3=} 100 \mathrm{mM} \mathrm{GB}+1.5 \% \mathrm{~K}$ & $1.9 \mathrm{a}$ & $5040.5 \mathrm{a}$ & $5784.0 \mathrm{a}$ \\
\hline $\mathrm{T}_{3} \mathrm{~K}_{0}=150 \mathrm{mM} \mathrm{GB}+0 \% \mathrm{~K}$ & $1.7 \mathrm{i}$ & $3930.8 \mathrm{~h}$ & $4500.5 \mathrm{fg}$ \\
\hline $\mathrm{T}_{3} \mathrm{~K}_{1}=150 \mathrm{mM} \mathrm{GB}+0.5 \% \mathrm{~K}$ & $1.7 \mathrm{gh}$ & $4395.8 \mathrm{f}$ & $4876.7 \mathrm{e}$ \\
\hline $\mathrm{T}_{3} \mathrm{~K}_{2=}=150 \mathrm{mM} \mathrm{GB}+1 \% \mathrm{~K}$ & $1.8 \mathrm{ef}$ & $4721.2 \mathrm{~d}$ & $5214.2 \mathrm{~cd}$ \\
\hline $\mathrm{T}_{3} \mathrm{~K}_{3=} 150 \mathrm{mM} \mathrm{GB}+1.5 \% \mathrm{~K}$ & $1.8 \mathrm{de}$ & $4869.7 \mathrm{bc}$ & $5574.0 \mathrm{~b}$ \\
\hline V x T, V x K, V x T x K & Non signif & & \\
\hline
\end{tabular}


Table 6. Average Water potential (-Mpa) of wheat leaves influenced by the application of glycinebetaine (GB) and potassium $(\mathrm{K})$ under drought in natural conditions. Means with different letters are significantly different from each other at $p \geq 0.05$.

\begin{tabular}{|c|c|c|c|}
\hline \multirow[t]{2}{*}{ Factors } & \multicolumn{3}{|c|}{ Experiments } \\
\hline & Pots trial & Field trial 2009-10 & Field trial 2010-11 \\
\hline $\mathrm{V}_{1}=$ Lasani-2008 & $0.80 \mathrm{~b}$ & $0.77 \mathrm{~b}$ & $0.75 \mathrm{~b}$ \\
\hline $\mathrm{V}_{2}=$ Auqab -2000 & $0.85 \mathrm{a}$ & $0.84 \mathrm{a}$ & $0.81 \mathrm{a}$ \\
\hline \multicolumn{4}{|l|}{ GB application rate $(\mathrm{T})$} \\
\hline $\mathrm{T}_{0}=0 \%$ & $1.09 \mathrm{a}$ & $1.06 \mathrm{a}$ & $1.00 \mathrm{a}$ \\
\hline $\mathrm{T}_{1}=50 \mathrm{mM}$ & $0.75 \mathrm{c}$ & $0.73 \mathrm{c}$ & $0.72 \mathrm{c}$ \\
\hline $\mathrm{T}_{2}=100 \mathrm{mM}$ & $0.63 \mathrm{~d}$ & $0.62 \mathrm{~d}$ & $0.59 \mathrm{~d}$ \\
\hline $\mathrm{T}_{3}=150 \mathrm{mM}$ & $0.82 \mathrm{~b}$ & $0.80 \mathrm{~b}$ & $0.78 \mathrm{~b}$ \\
\hline \multicolumn{4}{|l|}{$\mathrm{K}$ application rate $(\mathrm{K})$} \\
\hline $\mathrm{K}_{0=}=0 \%$ & $0.92 \mathrm{a}$ & $0.91 \mathrm{a}$ & $0.88 \mathrm{a}$ \\
\hline $\mathrm{K}_{1}=0.5 \%$ & $0.86 \mathrm{~b}$ & $0.84 \mathrm{~b}$ & $0.80 \mathrm{~b}$ \\
\hline $\mathrm{K}_{2=}=1.0 \%$ & $0.77 \mathrm{c}$ & $0.75 \mathrm{c}$ & $0.69 \mathrm{c}$ \\
\hline $\mathrm{K}_{3}=1.5 \%$ & $0.74 \mathrm{~d}$ & $0.72 \mathrm{~d}$ & $0.72 \mathrm{~d}$ \\
\hline \multicolumn{4}{|l|}{$\mathrm{T} \times \mathrm{K}$} \\
\hline $\mathrm{T}_{0} \mathrm{~K}_{0}=0 \% \mathrm{~GB}+0 \% \mathrm{~K}$ & 1.18 & $1.16 \mathrm{a}$ & $1.11 \mathrm{a}$ \\
\hline $\mathrm{T}_{0} \mathrm{~K}_{1=} \% \mathrm{~GB}+0.5 \% \mathrm{~K}$ & 1.13 & $1.10 \mathrm{~b}$ & $1.03 \mathrm{~b}$ \\
\hline $\mathrm{T}_{0} \mathrm{~K}_{2=}=0 \mathrm{~GB}+1 \% \mathrm{~K}$ & 1.04 & $1.01 \mathrm{c}$ & $0.94 \mathrm{c}$ \\
\hline $\mathrm{T}_{0} \mathrm{~K}_{3}=0 \% \mathrm{~GB}+1.5 \% \mathrm{~K}$ & 1.01 & $0.98 \mathrm{c}$ & $0.92 \mathrm{c}$ \\
\hline $\mathrm{T}_{1} \mathrm{~K}_{0=} 50 \mathrm{mM} \mathrm{GB}+0 \% \mathrm{~K}$ & 0.85 & $0.84 \mathrm{e}$ & $0.84 \mathrm{e}$ \\
\hline $\mathrm{T}_{1} \mathrm{~K}_{1=}=50 \mathrm{mM} \mathrm{GB}+0.5 \% \mathrm{~K}$ & 0.78 & $0.76 \mathrm{f}$ & $0.74 \mathrm{f}$ \\
\hline $\mathrm{T}_{1} \mathrm{~K}_{2}=50 \mathrm{mM} \mathrm{GB}+1 \% \mathrm{~K}$ & 0.70 & $0.68 \mathrm{~h}$ & $0.66 \mathrm{~h}$ \\
\hline $\mathrm{T}_{1} \mathrm{~K}_{3}=50 \mathrm{mM} \mathrm{GB}+1.5 \% \mathrm{~K}$ & 0.67 & $0.66 \mathrm{~h}$ & $0.64 \mathrm{hi}$ \\
\hline $\mathrm{T}_{2} \mathrm{~K}_{0=}=100 \mathrm{mM} \mathrm{GB}+0 \% \mathrm{~K}$ & 0.75 & $0.76 \mathrm{f}$ & $0.72 \mathrm{fg}$ \\
\hline $\mathrm{T}_{2} \mathrm{~K}_{1}=100 \mathrm{mM} \mathrm{GB}+0.5 \% \mathrm{~K}$ & 0.66 & $0.66 \mathrm{~h}$ & $0.61 \mathrm{i}$ \\
\hline $\mathrm{T}_{2} \mathrm{~K}_{2}=100 \mathrm{mM} \mathrm{GB}+1 \% \mathrm{~K}$ & 0.56 & $0.55 \mathrm{i}$ & $0.55 \mathrm{j}$ \\
\hline $\mathrm{T}_{2} \mathrm{~K}_{3}=100 \mathrm{mM} \mathrm{GB}+1.5 \% \mathrm{~K}$ & 0.55 & $0.53 \mathrm{i}$ & $0.50 \mathrm{k}$ \\
\hline $\mathrm{T}_{3} \mathrm{~K}_{0}=150 \mathrm{mM} \mathrm{GB}+0 \% \mathrm{~K}$ & 0.91 & $0.89 \mathrm{~d}$ & $0.87 \mathrm{~d}$ \\
\hline $\mathrm{T}_{3} \mathrm{~K}_{1}=150 \mathrm{mM} \mathrm{GB}+0.5 \% \mathrm{~K}$ & 0.86 & $0.84 \mathrm{e}$ & $0.82 \mathrm{e}$ \\
\hline $\mathrm{T}_{3} \mathrm{~K}_{2}=150 \mathrm{mM} \mathrm{GB}+1 \% \mathrm{~K}$ & 0.78 & $0.77 \mathrm{f}$ & $0.73 \mathrm{fg}$ \\
\hline $\mathrm{T}_{3} \mathrm{~K}_{3}=150 \mathrm{mM} \mathrm{GB}+1.5 \% \mathrm{~K}$ & 0.74 & $0.73 \mathrm{~g}$ & $0.71 \mathrm{~g}$ \\
\hline $\mathrm{V} x \mathrm{~T}, \mathrm{~V} x \mathrm{~K}, \mathrm{~V} \times \mathrm{T} \times \mathrm{K}$ & Non signif & & \\
\hline
\end{tabular}


Table 7. Average osmotic potential (-Mpa) of wheat leaves influenced by the application of glycinebetaine (GB) and potassium $(\mathrm{K})$ under drought in natural conditions. Means with different letters are significantly different from each other at $p \geq 0.05$.

\begin{tabular}{|c|c|c|c|}
\hline \multirow[t]{2}{*}{ Factors } & \multicolumn{3}{|c|}{ Experiment } \\
\hline & Pots trial & Field trial 2009-10 & Field trial 2010-11 \\
\hline $\mathrm{V}_{1}=$ Lasani-2008 & $1.40 \mathrm{~b}$ & $1.35 \mathrm{~b}$ & $1.29 \mathrm{~b}$ \\
\hline $\mathrm{V}_{2}=$ Auqab -2000 & $1.46 \mathrm{a}$ & $1.41 \mathrm{a}$ & $1.35 \mathrm{a}$ \\
\hline \multicolumn{4}{|l|}{ GB application rate $(\mathrm{T})$} \\
\hline $\mathrm{T}_{0=}=0 \%$ & $1.65 \mathrm{a}$ & $1.59 \mathrm{a}$ & $1.51 \mathrm{a}$ \\
\hline $\mathrm{T}_{1}=50 \mathrm{mM}$ & $1.38 \mathrm{c}$ & $1.33 \mathrm{c}$ & $1.29 \mathrm{c}$ \\
\hline $\mathrm{T}_{2}=100 \mathrm{mM}$ & $1.28 \mathrm{~d}$ & $1.22 \mathrm{~d}$ & $1.15 \mathrm{c}$ \\
\hline $\mathrm{T}_{3}=150 \mathrm{mM}$ & $1.41 \mathrm{~b}$ & $1.36 \mathrm{~b}$ & $1.34 \mathrm{~b}$ \\
\hline \multicolumn{4}{|l|}{$\mathrm{K}$ application rate $(\mathrm{K})$} \\
\hline $\mathrm{K}_{0=}=0 \%$ & $1.49 \mathrm{a}$ & $1.44 \mathrm{a}$ & $1.38 \mathrm{a}$ \\
\hline $\mathrm{K}_{1}=0.5 \%$ & $1.45 \mathrm{~b}$ & $1.40 \mathrm{~b}$ & $1.34 \mathrm{~b}$ \\
\hline $\mathrm{K}_{2}=1.0 \%$ & $1.40 \mathrm{c}$ & $1.34 \mathrm{c}$ & $1.30 \mathrm{c}$ \\
\hline $\mathrm{K}_{3}=1.5 \%$ & $1.38 \mathrm{~d}$ & $1.33 \mathrm{~d}$ & $1.27 \mathrm{~d}$ \\
\hline \multicolumn{4}{|l|}{$\mathrm{T} \times \mathrm{K}$} \\
\hline $\mathrm{T}_{0} \mathrm{~K}_{0}=0 \% \mathrm{~GB}+0 \% \mathrm{~K}$ & $1.71 \mathrm{a}$ & $1.66 \mathrm{a}$ & $1.55 \mathrm{a}$ \\
\hline $\mathrm{T}_{0} \mathrm{~K}_{1}=0 \% \mathrm{~GB}+0.5 \% \mathrm{~K}$ & $1.67 \mathrm{~b}$ & $1.61 \mathrm{~b}$ & $1.52 \mathrm{~b}$ \\
\hline $\mathrm{T}_{0} \mathrm{~K}_{2}=0 \% \mathrm{~GB}+1 \% \mathrm{~K}$ & $1.62 \mathrm{c}$ & $1.56 \mathrm{c}$ & $1.49 \mathrm{c}$ \\
\hline $\mathrm{T}_{0} \mathrm{~K}_{3}=0 \% \mathrm{~GB}+1.5 \% \mathrm{~K}$ & $1.61 \mathrm{c}$ & $1.55 \mathrm{c}$ & $1.47 \mathrm{c}$ \\
\hline $\mathrm{T}_{1} \mathrm{~K}_{0=} 50 \mathrm{mM} \mathrm{GB}+0 \% \mathrm{~K}$ & $1.43 \mathrm{e}$ & $1.38 \mathrm{~d}$ & $1.35 \mathrm{e}$ \\
\hline $\mathrm{T}_{1} \mathrm{~K}_{1}=50 \mathrm{mM} \mathrm{GB}+0.5 \% \mathrm{~K}$ & $1.41 \mathrm{f}$ & $1.35 \mathrm{f}$ & $1.30 \mathrm{~g}$ \\
\hline $\mathrm{T}_{1} \mathrm{~K}_{2}=50 \mathrm{mM} \mathrm{GB}+1 \% \mathrm{~K}$ & $1.36 \mathrm{~g}$ & $1.31 \mathrm{gh}$ & $1.28 \mathrm{gh}$ \\
\hline $\mathrm{T}_{1} \mathrm{~K}_{3=} 50 \mathrm{mM} \mathrm{GB}+1.5 \% \mathrm{~K}$ & $1.33 \mathrm{hi}$ & $1.29 \mathrm{~h}$ & $1.22 \mathrm{i}$ \\
\hline $\mathrm{T}_{2} \mathrm{~K}_{0=} 100 \mathrm{mM} \mathrm{GB}+0 \% \mathrm{~K}$ & $1.35 \mathrm{gh}$ & $1.31 \mathrm{gh}$ & $1.26 \mathrm{~h}$ \\
\hline $\mathrm{T}_{2} \mathrm{~K}_{1}=100 \mathrm{mM} \mathrm{GB}+0.5 \% \mathrm{~K}$ & $1.31 \mathrm{i}$ & $1.25 \mathrm{i}$ & $1.19 \mathrm{j}$ \\
\hline $\mathrm{T}_{2} \mathrm{~K}_{2}=100 \mathrm{mM} \mathrm{GB}+1 \% \mathrm{~K}$ & $1.23 \mathrm{j}$ & $1.17 \mathrm{j}$ & $1.09 \mathrm{k}$ \\
\hline $\mathrm{T}_{2} \mathrm{~K}_{3}=100 \mathrm{mM} \mathrm{GB}+1.5 \% \mathrm{~K}$ & $1.23 \mathrm{j}$ & $1.15 \mathrm{j}$ & $1.07 \mathrm{k}$ \\
\hline $\mathrm{T}_{3} \mathrm{~K}_{0}=150 \mathrm{mM}$ GB $+0 \% \mathrm{~K}$ & $1.46 \mathrm{~d}$ & $1.40 \mathrm{~d}$ & $1.39 \mathrm{~d}$ \\
\hline $\mathrm{T}_{3} \mathrm{~K}_{1}=150 \mathrm{mM} \mathrm{GB}+0.5 \% \mathrm{~K}$ & $1.43 \mathrm{e}$ & $1.38 \mathrm{de}$ & $1.35 \mathrm{e}$ \\
\hline $\mathrm{T}_{3} \mathrm{~K}_{2}=150 \mathrm{mM} \mathrm{GB}+1 \% \mathrm{~K}$ & $1.40 \mathrm{f}$ & $1.35 \mathrm{ef}$ & $1.33 \mathrm{ef}$ \\
\hline $\mathrm{T}_{3} \mathrm{~K}_{3}=150 \mathrm{mM} \mathrm{GB}+1.5 \% \mathrm{~K}$ & $1.37 \mathrm{~g}$ & $1.33 \mathrm{fg}$ & $1.30 \mathrm{fg}$ \\
\hline $\mathrm{V} \times \mathrm{T}, \mathrm{V} \times \mathrm{K}, \mathrm{V} \times \mathrm{T} \times \mathrm{K}$ & \multicolumn{3}{|c|}{ Non significant } \\
\hline
\end{tabular}


Table 8. Average Turgor potential (Mpa) of wheat leaves influenced by the application of glycinebetaine (GB) and potassium $(\mathrm{K})$ under drought in natural conditions. Means with different letters are significantly different from each other at $p \geq 0.05$.

\begin{tabular}{|c|c|c|c|}
\hline \multirow[t]{2}{*}{ Factors } & \multicolumn{3}{|c|}{ Experiment } \\
\hline & Pots trial & Field trial 2009-10 & Field trial 2010-11 \\
\hline $\mathrm{V}_{1}=$ Lasani-2008 & 0.60 & 0.57 & $0.56 \mathrm{~b}$ \\
\hline $\mathrm{V}_{2}=$ Auqab -2000 & 0.60 & 0.56 & $0.54 \mathrm{a}$ \\
\hline \multicolumn{4}{|l|}{ GB application rate $(\mathrm{T})$} \\
\hline $\mathrm{T}_{0}=0 \%$ & $0.56 \mathrm{~d}$ & $0.53 \mathrm{c}$ & $0.51 \mathrm{~b}$ \\
\hline $\mathrm{T}_{1}=50 \mathrm{mM}$ & $0.63 \mathrm{~b}$ & $0.59 \mathrm{a}$ & $0.56 \mathrm{a}$ \\
\hline $\mathrm{T}_{2}=100 \mathrm{mM}$ & $0.64 \mathrm{a}$ & $0.59 \mathrm{a}$ & $0.55 \mathrm{a}$ \\
\hline $\mathrm{T}_{3}=150 \mathrm{mM}$ & $0.59 \mathrm{c}$ & $0.56 \mathrm{~b}$ & $0.55 \mathrm{a}$ \\
\hline \multicolumn{4}{|l|}{$\mathrm{K}$ application rate $(\mathrm{K})$} \\
\hline $\mathrm{K}_{0=} 0 \%$ & $0.56 \mathrm{c}$ & $0.52 \mathrm{c}$ & $0.50 \mathrm{c}$ \\
\hline $\mathrm{K}_{1}=0.5 \%$ & $0.60 \mathrm{~b}$ & $0.56 \mathrm{~b}$ & $0.53 \mathrm{~b}$ \\
\hline $\mathrm{K}_{2}=1.0 \%$ & $0.63 \mathrm{a}$ & $0.59 \mathrm{a}$ & $0.57 \mathrm{a}$ \\
\hline $\mathrm{K}_{3}=1.5 \%$ & $0.64 \mathrm{a}$ & $0.60 \mathrm{a}$ & $0.57 \mathrm{a}$ \\
\hline \multicolumn{4}{|l|}{$\mathrm{T} \times \mathrm{K}$} \\
\hline $\mathrm{T}_{0} \mathrm{~K}_{0}=0 \% \mathrm{~GB}+0 \% \mathrm{~K}$ & $0.52 \mathrm{i}$ & $0.49 \mathrm{~h}$ & $0.45 \mathrm{~h}$ \\
\hline $\mathrm{T}_{0} \mathrm{~K}_{1}=0 \% \mathrm{~GB}+0.5 \% \mathrm{~K}$ & $0.55 \mathrm{~h}$ & $0.51 \mathrm{gh}$ & $0.49 \mathrm{gh}$ \\
\hline $\mathrm{T}_{0} \mathrm{~K}_{2=}=0 \mathrm{~GB}+1 \% \mathrm{~K}$ & $0.58 \mathrm{fg}$ & $0.55 \mathrm{ef}$ & $0.54 \mathrm{~d}-\mathrm{f}$ \\
\hline $\mathrm{T}_{0} \mathrm{~K}_{3}=0 \% \mathrm{~GB}+1.5 \% \mathrm{~K}$ & 0.59 ef & $0.57 \mathrm{de}$ & $0.56 \mathrm{~b}-\mathrm{e}$ \\
\hline $\mathrm{T}_{1} \mathrm{~K}_{0=} 50 \mathrm{mM} \mathrm{GB}+0 \% \mathrm{~K}$ & $0.58 \mathrm{fg}$ & $0.54 \mathrm{fg}$ & $0.51 \mathrm{fg}$ \\
\hline $\mathrm{T}_{1} \mathrm{~K}_{1}=50 \mathrm{mM} \mathrm{GB}+0.5 \% \mathrm{~K}$ & $0.63 \mathrm{~cd}$ & $0.60 \mathrm{bc}$ & $0.55 \mathrm{~b}-\mathrm{f}$ \\
\hline $\mathrm{T}_{1} \mathrm{~K}_{2}=50 \mathrm{mM} \mathrm{GB}+1 \% \mathrm{~K}$ & $0.66 \mathrm{ab}$ & $0.63 \mathrm{a}$ & $0.61 \mathrm{a}$ \\
\hline $\mathrm{T}_{1} \mathrm{~K}_{3}=50 \mathrm{mM} \mathrm{GB}+1.5 \% \mathrm{~K}$ & $0.66 \mathrm{ab}$ & $0.62 \mathrm{ab}$ & $0.58 \mathrm{a}-\mathrm{c}$ \\
\hline $\mathrm{T}_{2} \mathrm{~K}_{0=}=100 \mathrm{mM} \mathrm{GB}+0 \% \mathrm{~K}$ & $0.60 \mathrm{ef}$ & $0.55 \mathrm{ef}$ & $0.54 \mathrm{~d}-\mathrm{f}$ \\
\hline $\mathrm{T}_{2} \mathrm{~K}_{1}=100 \mathrm{mM} \mathrm{GB}+0.5 \% \mathrm{~K}$ & $0.65 \mathrm{bc}$ & $0.59 \mathrm{~cd}$ & $0.58 \mathrm{a}-\mathrm{d}$ \\
\hline $\mathrm{T}_{2} \mathrm{~K}_{2}=100 \mathrm{mM} \mathrm{GB}+1 \% \mathrm{~K}$ & $0.66 \mathrm{ab}$ & $0.62 \mathrm{ab}$ & $0.55 \mathrm{c}-\mathrm{f}$ \\
\hline $\mathrm{T}_{2} \mathrm{~K}_{3}=100 \mathrm{mM} \mathrm{GB}+1.5 \% \mathrm{~K}$ & $0.67 \mathrm{a}$ & $0.62 \mathrm{ab}$ & $0.57 \mathrm{~b}-\mathrm{e}$ \\
\hline $\mathrm{T}_{3} \mathrm{~K}_{0}=150 \mathrm{mM} \mathrm{GB}+0 \% \mathrm{~K}$ & $0.55 \mathrm{~h}$ & $0.51 \mathrm{~h}$ & $0.52 \mathrm{fg}$ \\
\hline $\mathrm{T}_{3} \mathrm{~K}_{1=} 150 \mathrm{mM} \mathrm{GB}+0.5 \% \mathrm{~K}$ & $0.57 \mathrm{gh}$ & $0.54 \mathrm{fg}$ & $0.53 \mathrm{e}-\mathrm{g}$ \\
\hline $\mathrm{T}_{3} \mathrm{~K}_{2=} 150 \mathrm{mM} \mathrm{GB}+1 \% \mathrm{~K}$ & $0.61 \mathrm{de}$ & $0.58 \mathrm{~cd}$ & $0.59 \mathrm{a}-\mathrm{c}$ \\
\hline $\mathrm{T}_{3} \mathrm{~K}_{3}=150 \mathrm{mM} \mathrm{GB}+1.5 \% \mathrm{~K}$ & $0.63 \mathrm{~cd}$ & $0.60 \mathrm{abc}$ & $0.60 \mathrm{ab}$ \\
\hline $\mathrm{V} \times \mathrm{T}, \mathrm{V} \times \mathrm{K}, \mathrm{V} \times \mathrm{T} \times \mathrm{K}$ & \multicolumn{3}{|c|}{ Non significant } \\
\hline
\end{tabular}


Analyzed data regarding leaf water potential (Table 6) showed that drought stress affected leaf water potential of both varieties. The lowest water potential was recorded in Lasani-2008 and the highest in Auqab-2000. Exogenous applications of GB under drought affected leaf water potential significantly. More negative leaf water potential was recorded in treatment $\mathrm{T}_{0}$, while less negative value was recorded with $T_{2}$ in pots as well as in field. Potassium also affected water potential significantly. Minimum value of water potential was recorded at $\mathrm{K}_{0}$, while maximum was observed at $\mathrm{K}_{3}(1.5 \%)$ both in pots as well as in field. Interactive effect of GB (T) and potassium (K), on water potential was non-significant in case of pots trial while significant in case of field trials during both years (Table 6). Negative water potential was maximum when crop was stressed without any spray (GB or $\mathrm{K}$ ) and the less negative water potential was obtained in $\mathrm{T}_{2} \mathrm{~K}_{3}$ which was similar to $\mathrm{T}_{2} \mathrm{~K}_{2}$ (Table 6).

Analyzed data regarding leaf osmotic potential (Table 7) showed that more negative leaf osmotic potential was recorded in Auqab-2000, while less negative was recorded in Lasani-2008. GB under drought significantly affected leaf osmotic potential; more negative leaf osmotic potential was recorded when crop faced water stress without GB spray $\left(\mathrm{T}_{0}\right)$ and less negative leaf osmotic potential was resulted in $\left(\mathrm{T}_{2}\right)$, (Table7). Effect of potassium spray levels on leaf osmotic potential was also recorded as significant. More negative leaf osmotic potential was recorded at $\mathrm{K}_{0}$ (no $\mathrm{K}$ under drought), while less negative was observed at $\mathrm{K}_{3}$ both in pots as well as in field (2009-10 and 201011) Interactive effect of exogenous applications of GB (T) and potassium (K), on osmotic potential was also significant in case of pots as well as in case of field trials (Table 7). Osmotic potential was more negative when crop was stressed at $\mathrm{T}_{0} \mathrm{~K}_{0}$ and the less negative osmotic potential was obtained in $\mathrm{T}_{2} \mathrm{~K}_{3}$ similar to $\mathrm{T}_{2} \mathrm{~K}_{2}$ (Table 7).

Leaf turgor potential (MPa) as indicated by analyzed data in Table 8 showed that leaf turgor potential was found non-significant on both varieties in case of pots and field trials (Table 8). Different levels of GB had significant effect on turgor potential. More leaf turgor potential was recorded in $T_{2}$ in pots trial, while in case of field trial same treatment was at par with 150 mM GB spray and less turgor potential was resulted under drought without GB application $\left(\mathrm{T}_{0}\right.$ ) (Table 8). Applications of $\mathrm{K}$ effect regarding leaf turgor potential indicated that it was maximum at $\mathrm{K}_{3}$ and $\mathrm{K}_{2}$ and minimum leaf turgor potential was resulted when crop had not received any exogenous applications of $\mathrm{K}\left(\mathrm{K}_{0}\right)$. Interactive effect of $\mathrm{GB}(\mathrm{T})$ and potassium $(\mathrm{K})$, on leaf turgor potential was also significant in case of pots as well as in case of field trials (Table 8). In pots, leaf turgor potential was maximum in $\mathrm{T}_{2} \mathrm{~K}_{3}$ and $\mathrm{T}_{2} \mathrm{~K}_{2}$, while in field it was maximum in $\mathrm{T}_{2} \mathrm{~K}_{3}$ and $\mathrm{T}_{2} \mathrm{~K}_{2}$ (Table 8). Minimum turgor potential was obtained in $\mathrm{T}_{0} \mathrm{~K}_{0}$ (Table 8).

\section{Discussion}

The results of our studies revealed that drought stress at grain filling affected the crop growth and development significantly by affecting all the yield components (spike length, number of spikelet's per spike, and number of grain per spike) and physiological parameters causing decline in the final grain yield of both the wheat varieties. This was in agreement with noticeably decrease in yield in the earlier studies in different crops as a result of water stress, e.g., in sugar beet (Bloch et al. 2006), wheat (Raza et al. 2102) and maize (Ashraf et al. 2007). Actually, the drought stress initiates a series of biochemical and physiological processes in plants which results in the reduction of crop yield (Shahbaz et al. 2011). Under these conditions a lot of adaptive mechanisms develop in plants to maintain productivity under adverse conditions. This includes the conservation of water by increase in root penetration, partial closing of stomata and reduction in transpiration (Hoad et $a l, 2001)$. Accumulation of solutes like GB and $\mathrm{K}$ in plants is one of such adaptive techniques to cope with drought stress by increasing the tolerance of plants and ameliorating the drought effects on growth and 
development of plant. In our study, foliar application of both solutes to wheat under water deficit condition significantly improved crop growth and development (plant height, spike length, number of spikelets per spike, number of grains per spike, and grain yield) and crop water relations (water potential, osmotic potential and turgor potential) during both years (2009-10 and 2010-11). This is corroborated with earlier studies (e.g. Raza et al. 2012, 2013) who reported drought tolerance of crop due to foliar applied solutes.

Exogenous application of both GB and $\mathrm{K}$ solutes to wheat under water deficit condition significantly affected the biochemical i.e., transpiration and physiological process i.e., photosynthesis and protein synthesis. GB is an effective osmoprotectant which naturally accumulates in a number of plants under drought stress (Zhang et al., 2009) thereby playing a vital role in drought tolerance of plants. However, it's not produced in ample amount to completely tolerate the effects of drought and thus need to be introduced in the plants. It has been reported in earlier studies that exogenous application of GB protects photosynthetic machinery by (i) preventing photoinhibition, (ii) preserving the net photosystem-II efficiency, and (iii) enhancing the tolerance of photosynthetic apparatus of wheat plants subjected to various stress conditions (Zhao et al. 2001; Demiral and Turkan, 2006). Consequently, in our experiments, application of GB significantly improved all the yield components of wheat, as compared to the wheat under stress without GB application. This beneficial effects of GB has been reported in a number of studies for various crops, particularly those which do not normally accumulate significant amount of GB under drought stress (e.g. Wang et al. 2010a; 2010b).

Foliar application of $\mathrm{K}$ to wheat under water deficit condition significantly improved the yield attributes and also affected water potential, osmotic potential and turgor potential of both wheat cultivars.
Combined spray of $100 \mathrm{mM} \mathrm{GB}$ and $1.5 \% \mathrm{~K}$ reduced the negative effect of drought on water relations of wheat plant and improved the tolerance of the plant under water deficit. These solutes also play a role as osmo-protectant by protecting cellular components from dehydration injury. Due to lower leaf water potential under drought stress biosynthesis of GB enhanced which finally improved its concentration in leaf (Zhu 2002). Exogenous application of GB in turnip rape plants improved net photosynthesis and reduced photorespiration under drought and salt stress (Makela et al. 1998). Exogenous applications of $100 \mathrm{mM} \mathrm{GB}$ under water stress conditions improved the 1000-grain weight and number of grains per spike through increased photosynthesis by maintaining leaf turgor potential. GB improved water deficit tolerance of the plant by maintaining internal water balance (turgor pressure) (Makela et al. 1998). The GB also enhanced leaf turgor potential by anchoring enzymes, functional proteins and lipids and maintained thylakoid membrane and electron flow through it (Allakhverdiev et al. 2003). Potassium enhances enzymes function to maintain the metabolic activities of plant under drought stress conditions. Plant growth is restrained under drought stress due to osmotic and ionic effects; however, plants have the ability to develop certain mechanisms to cope stress conditions (Munns 2002). Under water stress condition also toxic ions accumulate causing osmotic imbalance. Due to accumulation of free amino acids proline is well known to occur in large quantity in plants under abiotic stress conditions, contributing to osmotic adjustment (Ketchum et al. 1991).

Response of both varieties (Lasani-2008 and Auqab-2000) to the exogenous application of GB and $\mathrm{K}$ under drought was different; it may be due to their genetic makeup. i.e., the ability to reduce rate of transpiration (Iqbal et al. 1999) since Lasani-2008 tolerated drought more than Auqab-2000. These findings corroborates with $\mathrm{Liu}$ and $\mathrm{Li}$ (2005) who reported that a high leaf RWC under water deficits in drought resistant genotypes of wheat as compared to the drought sensitive. 


\section{Conclusions}

Results of this study revealed that drought stress at grain filling stage of wheat adversely affects its yield and physiological parameters. Foliar application of $\mathrm{GB}$ and $\mathrm{K}$ to the drought stressed wheat plants can help to improve water relations and the grain yield. Interesting, we found significant interactions between $\mathrm{GB}$ and $\mathrm{K}$, irrespective to the wheat variety. The combine application of $\mathrm{GB}$ and $\mathrm{K}$ at $100 \mathrm{mM}$ and $1.5 \%$, respectively, provided the best results. All these findings lead us to conclude that for wheat crop under drought farmers should spray GB or K to minimize the negative effect of drought. The combination of both these solutes can provide the best results. This can have dual benefits: improving yield of wheat under drought and supply of K nutrient to plants. Depending on the farm infrastructure, a common hand-boom sprayer can easily be used on small land-holder farms and mechanical boom sprayers on large scale farms.

\section{Acknowledgements}

The financial support of this study provided by Higher Education Commission of Pakistan is gratefully acknowledged. We are equally indebted to Nuclear Institute for Agriculture and Biology for providing technical support.

\section{References}

Akhtar, M.E., Ahmad, Z.K.S., Bashir, K. 2002. Response of different wheat cultivars to potash application in two soil series of Pakistan. Asian J. Plant Sci. 5, 535-537.

Allakhverdiev, S.I., Hayashi, H., Nishiyama, Y., Ivanov, A.G., Aliev, J.A., Klimov, V.V., Murata, N., Carpentier, R. 2003. Glycinebetaine protects the D1/D2/Cytb559 complex of photosystem II against photo-induced and heat-induced inactivation. J. Plant Physiol. 160, 41-49.
Allard, F., Houde, M., Krol, M., Ivanov, A., Huner, N. P. A., Sarhan, F. 1998. Betaine improves freezing tolerance in wheat. Plant Cell Physiol. 39, 1194-1202.

Ashraf, M., Foolad, M. R. 2007. Roles of glycinebetaine and proline in improving plant biotic stress tolerance. Enviro. Exp. Bot. 59, 206-216.

Bohnert, H.J., Nelson, D.E, Jensen, R.E. 1995. Adaptation to environmental stresses. Plant Cell.7, 1099-1111.

Demiral, T., Türkan, I. 2006. Exogenous glycinebetaine affects growth and proline accumulation and retards senescence in two rice cultivars under $\mathrm{NaCl}$ stress. Environ. Exp. Bot. 56, 72-79.

Evans, K.M., Riedell, W.E. 2006. Response of spring wheat cultivars to nutrient solutions containing additional potassium chloride. J. Plant Nutr. 29, 497-504.

Gadallah, M. M. A. 1999. Effects of proline and glycine betaine on Viciafaba responses to salt stress. Biol. Plant. 42, 249-257.

Govt. of Pakistan. 2012. Economic survey of Pakistan. Ministry of Food, Agriculture and Livestock, Finance Division, Economic Wing, Islamabad, Pakistan.

Hoad, S.P., Russell, G., Lucas, M.E, Bingham, I.J. 2001. The management of wheat, barley and oats root systems. Adv. Agron. 74, 193-246.

Iqbal, M., Ahmad, K., Sadiq, M., Ashraf, M.Y. 1999. Yield and yield components of durum wheat as influenced by water stress at various growth stages. Pak. J. Biol. Sci. 2, 11-14.

Ketchum, R.E.B., Warren, R.C., Klima, L.J., LopezGutierrez, F., Nabors, M.W. 1991. The mechanism and regulation of proline accumulation in suspension cultures of the halophytic grass Distichlisspicata L. J. Plant. Physiol. 137, 368-374. 
Liu, H.S., Li, F.M. 2005. Root respiration, photosynthesis and grain yield of two spring wheat in response to soil drying, Plant growth Regul. 46, 233-240.

Makela, P., Jokinen, K., Kontturi, M., Peltonen-Sainio, P., Pehu, E., Somersalo, S. 1998. Foliar application of glycinebetaine: a novel product from sugar beetas an approach to increase tomato yield. Indust. Crops Prod. 7, 139-148.

Mehdi, S. M., Ranjha, A. M., Sarfraz, M., Hassan, G. 2001. Response of wheat to potassium application in six soil series of Pakistan. J. Biol. Sci. 6, 429431.

Mengel, K., Arneke, W.W. 1982. Effect of potassium on the water potential, the pressure potential, the osmotic potential and cell elongation in leaves of Phaseolus vulgaris. Physiol. Plant. 54, 402-408.

Munns, R. 2002. Comparative physiology of salt and water stress. Plant cell. Environ. 25, 239-250.

Naidu, B.P. 1998. Simultaneous estimation of sugars, polyols, proline analogues and betaines accumulating in stressed plants by high performance liquid chromatography-Ultra violet detection. Aust. J. Plant Physiol. 25, 793-800.

Rahman, S., Miyake, M.H., Takeoka, Y. 2002. Effects of exogenous glycinebetaine on growth and ultrastructure of salt stressed rice seedlings (Oryza sativa L.). Plant Prod. Sci. 5, 33-44.

Raza, M.A.S., Saleem, M.F., Khan, I.H., Jamil, M, Ija, M,. Kha, M.A. 2012. Evaluating the drought stress tolerance efficiency of wheat (Triticum aestivum L.) cultivars. Russian J. Agric.SocioEconomic Sci. 12, 41-46.

Raza, M.A.S., Saleem, M.F., Shah, G.M., Jamil, M., Kha, I.H. 2013. Potassium applied under drought improves physiological and nutrient uptake performances of wheat (Triticum aestivum L.). J. Soil Sci. Plant Nutr. 13(1): 175-185.
Sakamoto, A., Murata, N. 2002. The role of glycine betaine in the protection of plants fromstress: clues from transgenic plants. Plant Cell Environ. 25, 163-171.

Serraj, R., Sinclair, T. R. 2002. Osmolyte accumulation: can it really help increase crop yield under drought conditions?. Plant Cell Environ. 25, 333-341.

Steel, R.G.D., Torrie, J.H., Dicky, D.A. 1997. Principles and procedures of statistics: A biometrical approach, 3rd edn. McGraw Hill, New York, USA.

Thalooth, A.T., El-Zeiny, H.A., Saad, A.O.M. 1990. Application of potassium fertilizer for increasing salt tolerance of broad bean (Viciafaba). Bulletin of Egypt Soc. Physiol. Sci. 10, 181-193.

Wang, G.P., Li, F., Zhang, J., Zhao, M.R., Hui, Z., Wang, W. 2010a. Overaccumulation of glycine betaine enhances tolerance of the photosynthetic apparatus to drought and heat stress in wheat. Photosynthetica. 48, 30-41.

Wang, G.P., Zhang, X.Y., Li, F., Luo, Y., Wang, W., 2010b. Overaccumulation of glycinebetaine enhances tolerance to drought and heat stress in wheat leaves in the protection of photosynthesis. Photosynthetica. 48, 117-126.

Zhang, L.X., Li, S.X., Liang, Z.S. 2009. Differential plant growth and osmotic effects of two maize (Zea mays L.) cultivars to exogenous glycinebetaine application under drought stress. Plant Growth Regul. 58, 297-305. 
Zhao, Z., Chen, G., Zhang, C. 2001. Interaction between reactive oxygen species and nitric oxide in drought-induced abscisic acid synthesis in root tips of wheat seedlings. Aust. J. Plant Physiol. 28, 1055-1061.
Zhu, J.K. 2002. Salt and drought stress signal transduction in plants. Annu. Rev. Plants Physiol Plant Mol. Biol. 53, 247-273. 